\title{
Nursing Practices in Dementia Management and Care in Nepal
}

Krishna Prasad Pathak, Ph. D. ${ }^{1}$

\begin{abstract}
Nurses fell barriers in management and care of dementia patients. A purposive random sampling approach identified 44 Registered Nurses (RN). The Knowledge of RNs' with regard to the diagnosis, management and care of dementia was unsatisfactory on the aspects of practices and management barriers are presented with regard to the following issues: negative views of dementia, difficulty in diagnosing early stage dementia, acceptability of specialists and responsibility for extra issues, knowledge of dementias and ageing, less awareness of declining abilities and diminished resources to handle care, guidelines, awareness of epidemiology and advice giving, and communicating the diagnosis. The following paper outlines the problems and solutions that Nepalese nurses' in the community need to adopt in order to deal effectively with its diagnosis, care and management.
\end{abstract}

Key words: Dementia, nurses, caregivers (cares), elderly, (RNs) registered nurses.

\section{Background of the Study}

The human resources profession in Nepal is: as per 100000 population the number of psychiatrists are $0.13,0.27$ nurses and psychological providers 0.19 that doesnot meet WHO standardization (Jha \&Sapkota, 2013,WHO -Aims 2006). There are neither sufficient psychiatric trained nurses nor specialized dementia nurses in Nepalese hospitals.

Dementia is a challenging issue to manage and care due to its overlapping signs and symptoms as well as its chronic mental health condition. In 2013, the Royal College of Nursing $(\mathrm{RCN})$ reported that dementia is challenge for hospitals and around a quarter of hospital beds are occupied by dementia patients. In 2011, the RCN published five principles for improving dementia care in hospital settings, which were; environment (Dementia friendly and support independence and well being), hospitals (assessment and early identification), staffs (skilled staff with enough time to care), partnership(family, friends and caregivers can be recognition and assessment) and individualized care

1 Dr. Pathak is a lecturer of Health Education at Koteshwor Multiple Campus, Koteshwor, Kathmandu affiliated to T.U. 
plans(it will be person centered need, dignity, palliative care, individual support, nutrition, comfort and rehabilitation). At the end of life many people with dementia (two thirds) spend their final years in hospital (McCarthy et al. 1997).

Many researchers have shown that dementia patients die during the cure time in hospital due to its longevity of its caring process (Sampson et al. 2009). In another study Sampson et al. (2006) showed that, people with dementia receive less palliative care compared with similar individuals without dementia in UK hospitals. However, for dementia patients palliative care is even more necessary. Guidelines have been drafted to assist nursing homes to develop policies and practices in the end-of-life care for residents with dementia (Cahill, Doran and Watson, 2010).

Dementia increases the disability rate among the elderly and creates the problems for both caregivers and the person (Hsiu-Li Huang et al. 2013). The care of older age people with dementia is bound to increase in Nepali society-increased longevity and disintegration of joint families, etc. This is the emerging evidence that the dementia problem is gradually expanding as a future crisis and a national challenge in Nepal.

In Nepal, there is virtually no awareness of this dementia problem amongst the public, professionals and policy makers. The illness places a heavy burden on both the elderly patient and their relatives. It can be estimated that currently about 135,000 people would be suffering from some kind of dementia in Nepal (Jha \& Nidesh 2013). This figure is likely to double every 20 years. There is a need for educational programs and healthcare policies that help to increase awareness of dementia in Nepalese nursing practice, management and care thereby improve the care provided to the Nepalese people. The main objective was to identify the key concepts in dementia practice, management and care from the experiences of RNs in the Nepalese hospital.

\section{Methods}

A purposive random sampling approach identified 44 Registered Nurses (RN). RNs were given a self completed questionnaire in English version, which were distributed and collected by the nursing head of those hospitals after taking the permission of hospital administration. These two private hospitals were newly established with fifty beds in Kathmandu.

Most RNs were working as full timer in different wards. The respondent (RNs) was only 44 where around 90 nurses were working night and day shift asa full timer. Twenty answers were from one hospital and 24 answers was from next hospital. To distribute these questions was not any bias with RNs however, those who were interested to fill 
up, only for these were distributed by the head of nurse of those hospitals eventhough, 22 were returned as blank. Among of these blanked (from 22) were completed half and some were totally blank so for these questions were not included in this result.

The questionnaires prompted respondents answer a dementia quiz: concerning diagnosis, epidemiology, and management of dementia. It was on multiple choice items for each question as well as in the aspect of dementia quiz there was "do not know" option on each question. In first part 18 questions were included with multiple choices what was related to the current practices in dementia care with the nurses and the second part 7 questions were dementia epidemiology quiz, 2 questions were concerned with medication effects and its uses to the patients. Likewise, the one question was related to the multidimensional nature of dementia diagnosis. Another question concerned the prevalence of dementia and the last two questions were open and prompted respondents' to share their experiences of dementia care, management, and difficulties in their work.The research intension was to find the recently established private hospitals' RNs' knowledge, barriers and practices concerning nurses' management and care of dementia patients from whole nurses, but because of difficulties of private hospital rules and regulation to the staffs in their duty. These RNs was working in a variety of clinical care, including OPD, ICU/ OT ward, general ward, emergency ward, medical ward, gynecological ward, orthopedic ward, pediatric ward, psychiatric ward. All of these data are based on the RNs' responses. Most of these answered was lately returned from the (RNs) respondents.

The questionnaire format was similar with Turner's et al., (2004). These questions were coded according to the Statistical Package for the Social Sciences, SPSS version 20.

\section{Results}

The data has been presented on the basis of total 44 nurses from 2 private hospitals in Kathmandu, 2019. These RNs were worked as a full timer in a variety of clinical care, including ICU/OT ward, general ward, emergency ward, medical ward, gynecological ward, orthopedic ward, pediatric ward, psychiatric ward. In this result section data are presented under the following subtitles; knowledge of dementia, demographic characteristics, Dementia diagnosis practices in hospitals of the health professionals, Awareness of dementia care services, Obstacles to management and care, Obstacles to management and care.

The total mean score of participants' dementia care knowledge was very low. Overall, the Minimum correct answer was given 1 and maximum 7(Mean 3.54, SD.1.82). Forty four RNs' responses to the knowledge quiz are shown in table 1.in appendix A. 


\section{Demographic characterises}

This study did find differences with regard to either working experiences or age. However, the majority of nurses were young (Mean age was 24.04 SD =3.68). This study provided initial insights into the dementia care situation at hospital in Nepal. Future studies should further explore the relationship between dementia care quality and hospital nurses' knowledge and approaches accordingly.

\section{Dementia diagnosis practices in hospitals by health professionals}

The result shows that the most diagnostic practices are based on with physicians 16 $(36.4 \%)$ at the first clinical visit and the second part is with the neurologist $13(29.5 \%)$ than others professional doctors to the dementia patients. Accordingly, GP colleague 6 (13.6\%), old age psychiatric $5(11.4 \%)$, Geriatrician $13(29.5 \%)$ is the sub diagnostic role on the basis of RNs expectation. These RNs reported that to diagnose the dementia patients it may take one month to sixteen months for the suspected dementia patients on their experiences. However, thirty four (77.3\%) RNs reported "do not know" the time period of diagnosis in the clinical process. As well as $30 \mathrm{RNs}(68.2 \%)$ reported that not able to say the exact number of dementia patients in a typical month. However, In general 1-2 patients had been visited and diagnosed monthly in their experiences.

This study revealed that $16(36.4 \%)$ RNs reported that a behavioral problem was mostly diagnosed with the suspected demented patients by the doctors as a witness. Accordingly, Parkinson disease, Alzheimer diseases, Huntington disease were declared in their hospital.

Majority RNs 24 (54.5\%) was suggested to visit the a team of psychologist and multi expert doctors for the dementia loved ones (parents or for their relatives) in their practice. Additionally, 4 (9.1\%) general practitioners, Neurologist 7 (15.9\%), Geriatrician 6 (13.6\%) were suggested to visit the above concerned doctors by the RNs.

This study revealed that $16(36.4 \%)$ RNs reported that behavioral problems were diagnosed with the suspected demented patients. Accordingly, Parkinsons disease, Alzheimer diseases, Huntington disease were declared in their hospital. Our data show the majority RNs $24(54.5 \%)$ were suggested to consult the psychologist and primary care doctors to detect dementia issue for their patients and their relatives than other health professionals. Similarly, 4(9.1\%) general practitioners, neurologist 7(15.9\%), Geriatrician 6(13.6\%) were suggested to visit the above concerned doctors by RNs.

Result showed that the lowest dementia quiz score was 4 (9.1\%) and the highest quiz score was $30(68.2 \%)$. So it shows that the knowledge is very poor with the RNs. So dementia care and management is not fully established in hospital settings. 


\section{Awareness of dementia care services}

In the aspect of dementia- memory clinic services, dementia local supported services, clinical services, availability of medicine was very low, one third RNs were unknown regarding with these entire sectors. In the aspects of local support of dementia very few 18 nurses (40.9\%) were unknown, as the same way they were confirmed that there was no any local support to the demented patients $(40.9 \%)$, however only $3(6.8 \%)$ could name memory clinic and location. Similarly 27 (61.4\%) knew that there was day care service. This result prooved that awareness is important to the nurses even though they all were full time worker in the hospital.

There was another measurement scale to assess the RNs' awareness regarding with the availability of dementia related medicine. As per our data majority RNs12 (27.35\%) were unknown regarding with dementia medicine, which was asked of them in a questionnaire 'Is there available dementia medicine in the market or not'. Likewise, $10(22.7 \%)$ replied was very difficult to find and remaining $9(20.5 \%)$ answered No(means not available), yes(means available) $8(18.2 \%)$ is available in the market. Above these data clearly indicated that the dementia is burdensome disease, but still not much prioritized public health issue with the health professionals than other disease in hospital care neither they were more alert to the dementia issue.

\section{Obstacles to management and care}

Not only GPs, caregivers, family members has problematic the dementia, it has problematic to the nurses for management and care in the hospital settings also. In this study, RNs indicated that the key point of the obstacle factors to manage and care for dementia patients, such age;1) disbelief, 2) mis-diagnosis, 3) late referral, 4) lack of knowledge and 5) high cost.

That's why they (RNs) suggested taking advantage from the early diagnosis. And also they were not satisfied with the current practice which has done in their hospital practice in the present context. There was high rank of an unsatisfactory number of RNs 16 (36.4\%) than satisfied number of RNs 5 (11.4\%). Even though there were another significant number of RNs 20 (45.5\%) who were unknown either it is (current practices regarding with dementia) appropriate or not.

\section{Discussion}

We examined dementia knowledge, practices, management and care in nursing practices with Nepalese RN. This study also highlights that Alzheimer's dementia disease is considerably less $8(18.2 \%)$ diagnosed with the Nepalese population than other types of disease in their experiences, therefore it is increasing evidence that it is 
a commonly missed diagnosis in older people by health professionals. In the US, 5.2 million have $\mathrm{AD}$, and it isrecognized as the sixth leading cause of death among older people(Alzheimer Association, 2013). In Nepal there is virtually no awareness of the dementia problem amongst the public, professionals and policy makers. Even if it is not recognized as dementia, the illness places a heavy burden for the both elderly patients and their relatives. It can be estimated that currently about 135,000 people would be suffering from some kind of dementia in Nepal (Jha\& Sapkota, 2013).

In our study nursing care and management confident was poorer than in Taiwan, where community health nurses were confident to care and management of dementia positively, because nursing practice with the ageism is not by professional interests, led by the views of older people (Jan R., et al. 2012). Still now the major trend is to care for dementia patients by relatives or spouse at home (Zarit et al. 1999; Hsiu-Li H. et al. 2013). Without doubt, the majority of carers felt that a key role goes to the GP.

In one study, only $47.6 \%$ of GPs agreed they had a sufficient training chance in the management and diagnosis of dementia and early diagnosis as well as $54.4 \%$ felt, it was significant to look objectively for early signs of dementia. Similarly, in our study also the nurses were reported to take the early diagnosis to of the suspected patients, but they had very low chance to take the raining of management and care (Judy R. et al. 2000). Understanding the social burden issue of dementia and its costs is crucial for future health care and socioeconomic policymakers as well as should address to the under detection cause and treatment procedure, by making strategies to increase awareness and training for health professionals, public media and to the community (Raj K.N.et al. 2008).Above all these data also refer the dementia problem is burdensome diseases, but still not so much prioritized public health issue with the health professionals. This should support better planning and delivery of services.

In our study, RNs indicated that the key obstacles to managing and dementia were; 1) disbelief, 2) mis -diagnosis, 3) late referral, 4) lack of knowledge and 5) high cost as well as. In the aspect of dementia, the memory clinic services, dementia local supported services, clinical services, availability of medicine was very low, one third RNs were unknown regarding with these entire sectors.

Nurses feel insufficiently prepared to provide appropriate services and would take no action on suspected dementia. The study concluded that nurses would benefit from improved training in this area and that this could lead to improved access to services for people with dementia and their carers. At last,the findings suggested the need for educational programs and health care policies that increase awareness of dementia in nursing practice and thereby improve the care provides to people with dementia. 


\section{Recommendations}

Nepalese RNs need to have specific dementia assessment tools/guidelines for better care and management. RNs have a crucial part to play for better diagnosis, monitoring and providing long term support for the dementia patients. In this regard, especially in Nepal's RNs case, they need to work more closely with the caregivers, geriatricians, neurologists, social workers, NGO/INGOs; allied health teams and psychiatric hospital. Many of the aforementioned issues can be addressed via, their professional educational development. More priority needs to be focused towards; quality development, research and planning, active care, improving collaboration with professional doctors and medical follow up, understanding and responding to behavior changes, care and management with screening instruments. Efforts in Nepal need to be directed towards improving financial support, community support, family support, establishing day care home services, memory clinic services with geriatric services, a separate department of dementia in the hospital and in the community. Improvements in dementia knowledge and self-confidence for management and care should lead to an improvement in service delivery in a Nepalese hospital with nurses.

\section{References}

Alzheimer's disease facts and figures (2013). Alzheimer's Association / Alzheimer's \& Dementia 9; 208-245.

Azermai M, Kane J, Liperoti R, Tsolaki M, Landi F, Passmore AP, Petrovic M, \& CruzJentoft A. (2013).Management of behavioral and psychological symptoms of dementia: Belgium, Greece, Italy, United Kingdom. European Geriatric Medicine 4 (2013) 50-58.

Barker S (2007) .Vital notes for nurses: psychology, Wiley-Blackwell.

Cahill, S., Doran, D., \& Watson, M. (2012). Guidelines for nursing homes delivering end-of-life care to residents with dementia across the island of Ireland. Quality in Ageing and Older Adults (13) 1, 60-71. 2.

Department of health services (2011). Annual report. Government of Nepal, ministry of health and population.

Hendrie, C.H, Frederi, C.H \& Unverzagt W(1997). The dementing disorders. PsychiatricQuarterly Vol.68, no.3.

Hsiu-Li H, Yea-Ing S.L, Huei-Ling H, Sien-Tsong C,Hsiao-Juan L, Ming-Neng S, Mei-Chi P(2013). Factors associated with dementia care practices among community health nurses: Results of a postal survey.International Journal of Nursing Studies 50: 1219-1228. 
Jha A \& Sapkota N (2013). Dementia Assessment and Management Protocol for Doctors in Nepal. Journal of Nepal Medical Association 52(189):292-8.

Kalaria R.N, Maestre GE, Arizaga R., Friedland R P, Galasko D, Hall K., Luchsinger JA, Ogunniyi A, Perry E K, Potocnik F, Prince M, Stewart R., Wimo A, Zhang Zhen-xin, Antuono P $\&$ for the World Federation of Neurology Dementia ResearchGroup(2008). Alzheimer's disease and vascular dementia in developing countries: prevalence, management, and risk factors. The lancet Neurology 7, Issue 9, 812-826.

McCarty M, Addington-Hall J, \& Altmann, D (1997). The experience of dying with dementia: a retrospective study. International Journal of Geriatric Psychiatry 12: 404-9.

Reed J, Clarke C. \& Macfarlane A. (2012). Nursing older adults. World wideweb: www. openup.co.uk. ISBN-13: 978-0-335-24084-5; ISBN-10: 0-335-24084-4, eISBN: 978-0-335-24085-2. Open University Press, McGraw-Hill Education, McGrawHill House, Shoppenhangers Road, Maidenhead, Berkshire, England, SL6 2QL.

Renshaw J, Scurfield P, Cloke Luise \& Orrell M (2000). General practitioners' views on the early diagnosis of dementia. British Journal of General Practice51, 37-38.

Sampson E.L, Blanchard M.R, Tookman J L A \& King, M (2009) Dementia in the acute hospital:prospective cohort study of prevalence and mortality', British Journal of Psychiatry 195(1): 61-6.

Sampson, E.L, Gould, V, Lee, D \& Blanchard, M.R (2006). Differences in care received by patients with and without dementia who died during acute hospital admission: a retrospective case note study, Age and Ageing 35: 187-9.

Thompson, R \& Heath H (2013). Dementia: commitment to the care of people with dementia in hospital settings. www.rcn.org.uk/dementia.

Waldemar G, Dubios B, Emre M, Scheltens P., Tariska P \& Rosser M (2000). Diagnosis and management of Alzheimers disease and other disorders associated with dementia. The role of neurologist in Europe. European journal of Neurology 133-144.

WHO-AIMS(2006). Report on Mental Health System in Nepal, WHO and Ministry of Health, Kathmandu, Nepal.

Zarit SH, Gaugler JE, Jarrott SE. (1999) .Useful services for families: research, Endings and directions. International Journal of Geriatric Psychiatry 14: 165-181. Nepal nursing council.www.nnc.org.np. 